J3eA, Journal sur l'enseignement des sciences et technologies de l'information et des systèmes, Volume 2, Hors-Série 2, 15 (2003)

DOI : http://dx.doi.org/10.1051/bib-j3ea:2003615

(C) EDP Sciences, 2003

Problèmes de l'intégration de la puissance et de la transmission de données dans une capsule

R. Duffait* et L. Boillod**

* C.T.M.

39 avenue de l'Observatoire

F-25007 Besançon cedex 3, France

** ISA-France S.A.

8 chemin des Clos-Rondot

F-25130 Villers-Le-Lac, France

roland.duffait@ctm-France.com, laurent.boillodeisa-fr.com 


\section{Problèmes de l'intégration de la puissance et de la transmission de données dans une capsule intestinale}

\author{
Roland Duffait \\ C.T.M. \\ 39, av. de l'Observatoire, \\ 25007 Besançon cedex 3 \\ roland.duffait@ctm-France.com
}

\author{
Laurent Boillod \\ ISA-France S.A. \\ 8, chemin des Clos-Rondot \\ 25130 Villers-Le-Lac
}

laurent.boillod@isa-fr.com

\section{Résumé}

Sur la base d'un concept de modularité et en utilisant l'expérience de l'Hôpital Civil de Strasbourg, un micro dispositif à usage unique, destiné à l'investigation de l'intestin grêle, a été développé sous l'impulsion d'un projet franco-suisse du programme européen INTERREG II. Ce projet, coordonné par le CTM et la Fédération Suisse pour la Recherche en Microtechnique (F.S.R.M.), réunit l'Hôpital Civil de Strasbourg, l'Institut des Microtechniques de FrancheComté, des écoles d'ingénieurs et les universités de Franche-Comté et des cantons de Neuchâtel et de Vaud ainsi que des partenaires industriels français et suisses.

\section{La capsule intestinale: une solution intéressante}

L'intestin grêle est un organe essentiel dans le processus de digestion des aliments, mais il est également le siège de bactéries et de parasites susceptibles de provoquer des maladies. Pour le diagnostic de ces dernières les moyens classiques d'investigation ne permettent pas de l'explorer sur toute sa longueur: environ cinq à sept mètres sont inaccessibles aux méthodes endoscopiques. L'idée d'un dispositif autonome se déplaçant sous l'action du transit intestinal a été développée par Alain Lambert, ingénieur à l'INSERM (Institut National pour la Santé et la Recherche Médicale) et par le Professeur Jacques F. Grenier, chirurgien à l'Hôpital Civil de Strasbourg [1]. Avec leur collaboration nous avons ensuite repris ces travaux dans le cadre d'un projet franco suisse du programme européen INTERREG II. Outre ses possibilités d'exploration de tout l'intestin grêle, ce dispositif présente l'avantage d'un plus grand confort $\mathrm{du}$ patient, qui n'a plus besoin, ni de subir une anesthésie, ni même d'être immobilisé.

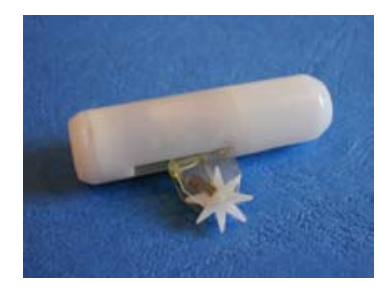

Figure 1 . Capsule intestinale originale de Strasbourg
La capsule d'origine développée à Strasbourg est composée d'un module de base comportant la source d'énergie, un dispositif de détection de la distance parcourue et du sens de déplacement dans l'intestin, une électronique pour la transmission de ces informations vers le PC du praticien. La mesure de la distance parcourue est particulièrement simple et donne de très bons résultats. Elle se présente sous la forme d'une roue qui se déploie lorsque la capsule se trouve dans l'estomac et qui délivre des impulsions à intervalles réguliers, comptées par l'électronique. Des modules fonctionnels, montés sur le corps de base suivant les besoins, assurent des fonctions de largage d'un médicament, de prélèvement de contenu ou de paroi intestinale. Le déclenchement de ces fonctions est assuré par l'approche d'un aimant vers l'abdomen du patient.

\section{Autres réalisations dans le monde}

Parmi les réalisations remarquables les plus récentes, il faut citer la capsule réalisée par le M.I.T. (Massachussetts Institute of Technology) de J. Santini, M. Cinna et R. Langer [2], le module-caméra de la société israëlienne Given Imaging et dernièrement, la pilule-Norika de l'institut japonais RF System Lab [3]. Le dispositif du MIT n'est destiné qu'au largage de médicaments. Il se compose d'une pastille de silicium comportant des micro alvéoles refermées chacune par un opercule d'or servant d'anode. Chaque alvéole contient quelques micro litres de principe actif qui est libéré par la dissolution de la pellicule d'or sous l'effet d'un faible courant. Le système de Given Imaging est une micro caméra insérée dans une pilule. Les images sont transférées à un ensemble de mémoires disposées dans une ceinture portée par le patient. La pilule Norika permet tout à la fois l'imagerie, un largage de substance active et un prélèvement de liquide intestinal.

\section{Dispositif INTERREG}

\subsection{Cahier des charges}

Dans le but de l'extension des possibilités de la capsule de Strasbourg, nous nous sommes fixés les objectifs suivants :

- Usage unique,

- Possibilité de répétition des fonctions de largage ou de prélèvement au cours d'un même transit, 
- Modularité complète au niveau de la chaîne de fabrication, quel que soit le module fonctionnel monté, - Mesure de paramètres internes tels que la température, la pression ou le $\mathrm{pH}$,

- Extension des fonctions électroniques : traitement et transmission des données et des ordres, signal de fin de fonction, électronique de puissance pour le pilotage du module moteur,

- Portée de l'émetteur-récepteur : quatre à cinq mètres,

- Durée de vie : 24 heures pour tenir compte des durées possibles de stationnement dans l'estomac et du transit intestinal,

- Volume total de principe actif embarqué : environ $1 \mathrm{~cm}^{3}$,

- Dimensions totales de la capsule, imposées par le corps médical : diamètre $11 \mathrm{~mm}$. Longueur inférieure ou égale à $40 \mathrm{~mm}$.

- Consommation totale inférieure à $10 \mathrm{~mA}$

- Durée maximum d'exécution des fonctions : 10 secondes

\subsection{Architecture générale}

Les principes de modularité et de répétitivité des fonctions nous ont imposé le schéma général de la capsule (figure 2): Le module de base comprend l'électronique commune à toutes les fonctions : émetteur-récepteur, micro contrôleur pour le traitement des ordres et des données, interfaces de connexions. Les modules fonctionnels comprennent à l'heure actuelle le largage répété de plusieurs substances liquides, pulvérulentes ou solides et le prélèvement prélèvements répétés de contenu intestinal. Le module d'entraînement est composé d'un micro moteur de type horloger associé à un engrenage réducteur. Le système de localisation de la capsule est basé sur le principe de l'Hôpital de Strasbourg. Par contre ce dernier envoie au $\mathrm{PC}$ récepteur la valeur absolue de la distance parcourue et le sens de déplacement. Contrairement à la capsule de Strasbourg, ces données ne sont plus calculées par le $\mathrm{PC}$ récepteur.

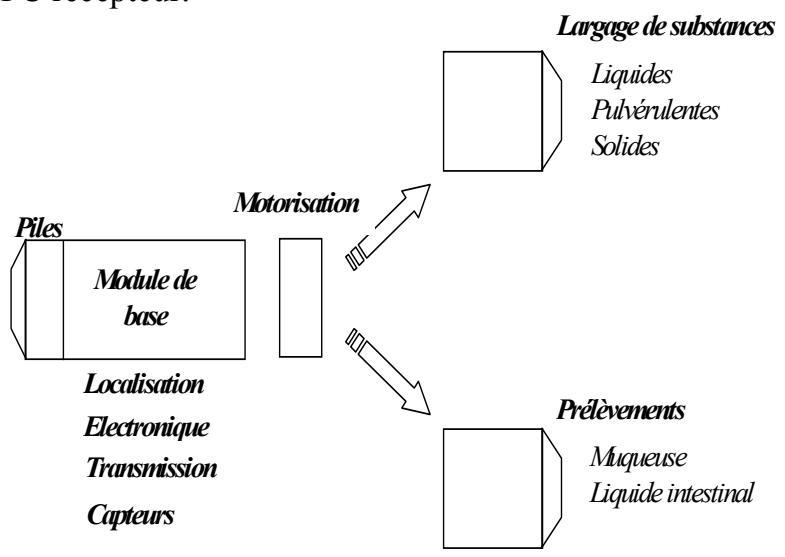

Figure 2. Architecture générale de la capsule Interreg II

Le choix des solutions est guidé par un triple objectif de consommation minimum, de réduction de l'encombrement pour libérer le maximum de volume utile aux substances embarquées et de limitation des coûts de fabrication. Le premier concerne non seulement les organes de puissance et de transmission des données, mais également les modules fonctionnels mécaniques situés en aval de la chaîne cinématique. Le dernier point n'est pas forcément compatible avec les deux premiers.

\section{Choix de l'éléments moteur}

\subsection{Types de solutions envisagés}

Compte - tenu de ces spécifications, nous avons retenu deux voies d'investigation: l'utilisation d'alliages à mémoire de forme (AMF) et la réalisation d'un mécanisme de type horloger. Nous avons pris la décision de les développer simultanément toutes les deux jusqu'à ce qu'il soit possible de faire un choix indiscutable.

\subsection{Utilisation des alliages à mémoire de forme}

4.2.1 description générale. Cette solution paraissait à priori extrêmement simple: un fil en AMF, relié à un piston, se rétrécit sous l'action de la chaleur provoquée par le passage d'un courant. La contraction du fil permet la libération du principe actif (figure 3 ).

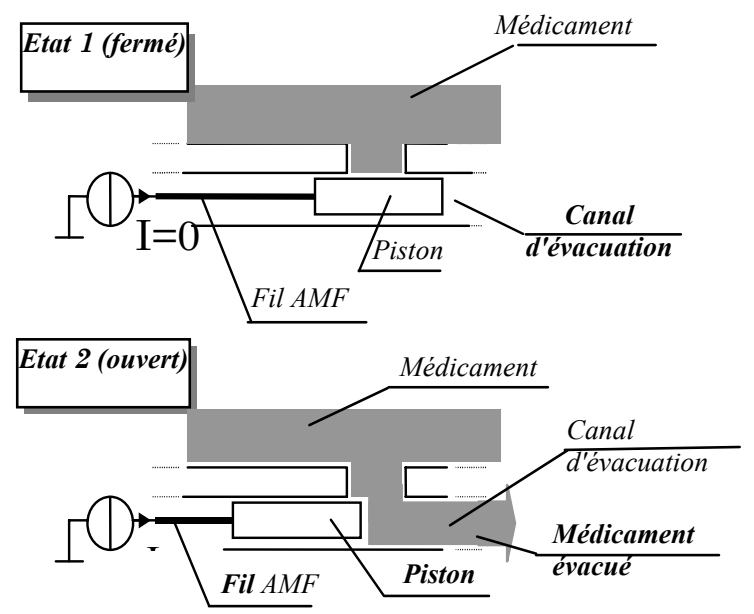

Figure 3. Dispositif à alliage à mémoire de forme

Pour limiter la consommation en courant, nous pouvions agir sur les paramètres suivants :

- Tension du fil : les températures de transformation des alliages à mémoire de forme dépendent de leur composition mais également des conditions dans lesquelles ils sont placés, telles que les contraintes qui leur sont appliquées. La tension des fils doit donc être définie avec précision.

- Ecart entre la température ambiante et la température de transformation du fil : il doit être aussi réduit que possible, moyennant quelques sécurités (par exemple pour tenir compte d'un éventuel état fébrile du patient au cours de l'essai).

-Diamètre du fil: pour une longueur de fil donnée la consommation de courant nécessaire à l'échauffement, varie avec le carré du diamètre du fil. Il s'agissait donc de choisir le fil le plus fin possible capable de supporter les contraintes de traction du piston.

Une seule société, basée en Californie, possédait un catalogue suffisamment complet pour pouvoir nous livrer un fil correspondant à nos besoins. 
4.2.2 Caractérisation. Elle a été réalisée en collaboration avec le Laboratoire de Mécanique Appliquée Raymond Chaléat de Besançon (L.M.A.R.C.), le Laboratoire d' Automatique de Besançon (L.A.B.) et l'Institut de Génie Energétique de Belfort (I.G.E.), tous ces laboratoires faisant partie de l'Université de Franche-Comté. Les essais ont été réalisés grâce à l'aide de deux spécialistes des alliages à mémoire de forme : M Ellal Benzahoui du L.A.B. et le professeur Christian Lexcellent, du LMARC. Il s'agissait, à partir d'une température ambiante donnée, de déterminer exactement la température de transition et l'apport d'énergie nécessaire à la transformation. Le montage d'essai mis au point dans ces laboratoires est représenté à la figure 4 ci-dessous :

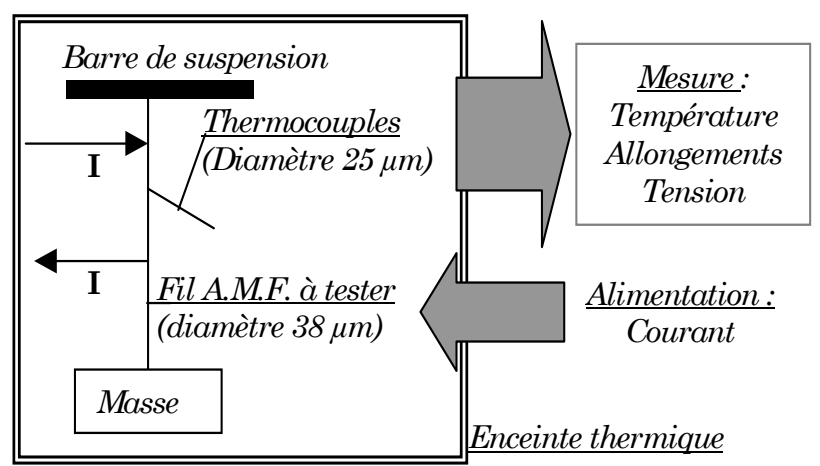

Capteur- laser

(mesure des allongements

Figure 4. Dispositif de caractérisation des fils AMF (LABLMARC

Le fil est suspendu dans une enceinte thermique dans laquelle la température ambiante est contrôlée. Il est maintenu en tension à l'aide d'un poids de valeur prédéterminée et alimenté en courant. La mesure de la température $\mathrm{du}$ fil est effectuée à l'aide de thermocouples miniaturisés d'un diamètre de $25 \mu \mathrm{m}$, réalisés et soudés sur le fil à tester par $M$ Thierry Laurent, de l'I.G.E. Les mesures effectuées sont de différents types:

- Variation en fonction du temps de la température du fil alimenté

- Variation de la résistivité du fil en fonction de la température

- Variations de longueur en fonction de la température

4.2.3 Résultats obtenus. Ces mesures ont permis de déduire avec exactitude les températures de transformation et la consommation en courant. Elle a permis d'établir qu'un courant de $30 \mathrm{~mA}$ était nécessaire à la transformation, sans tenir compte des pertes de chaleur dues aux différents raccordements. Etant donné la source d'énergie possible dans la capsule, ce résultat a été décrété comme rédhibitoire par les partenaires chargés de la micro électronique.

4.2.4 Conclusion. La réalisation d'un prototype fonctionnel nous a permis de constater que, outre la consommation excessive de courant, ce dispositif était difficile à monter en dépit de sa simplicité de fonctionnement. Les points sur lesquels nous avons dû tout particulièrement porter notre attention sont les suivants :

- Pertes calorifiques au niveau des raccordements électriques.

- Raccordement mécanique des fils, géométrie de l'enroulement pour ne pas perturber la contraction, réglage de la tension exacte du fil pour limiter les chemins perdus ou ne pas augmenter les températures de transformation.

- Protection contre les risques de déclenchement intempestif à la suite d'une exposition à la chaleur (par exemple, après un séjour sur la plage arrière d'une voiture pendant le transport)

En conclusion, d'un principe au départ extrêmement séduisant, cette solution présentait des inconvénients au point de vue de la consommation électrique et du montage qui nous ont conduits à préférer le système de type moto réducteur, développé parallèlement par le C.T.M

\subsection{Solution de type moto réducteur}

4.3.1 Description générale. Ce dispositif utilise les techniques horlogères traditionnelles de la région FrancheComté. Les possibilités de production autorisent une fabrication à faible coût. Il comprend un moteur pas-à-pas de type Lavet (figure 5), qui équipe actuellement la quasi totalité des montres à quartz analogiques. Le moteur entraîne le mécanisme de déclenchement par l'intermédiaire d'un engrenage réducteur.

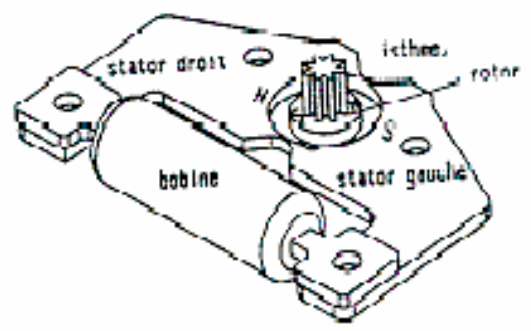

Figure 5. Perspective schématique du moteur Lavet

Le rotor se compose d'un aimant bipolaire monté sur l'axe du premier pignon d'entraînement du rouage. Il est placé dans l'entrefer d'un électroaimant constitué d'un stator en matériau à haute perméabilité magnétique et d'une bobine. Une dissymétrie des épanouissements polaires du stator permet de donner, au repos, une orientation de l'axe des pôles du rotor pour prédéfinir le sens de rotation du moteur (figure 6).

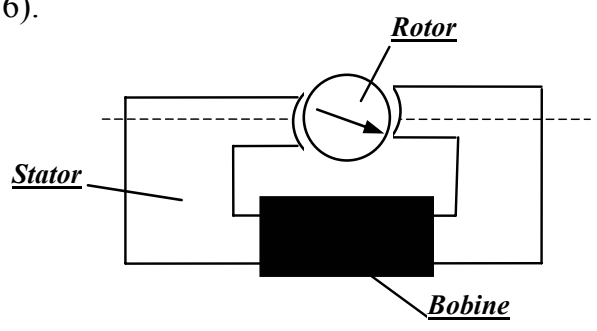

Figure 6. Shéma de principe du moteur Lavet

Le rotor effectue des pas d'une valeur angulaire de $180^{\circ}$. L'alternance de pôles nord et de pôles sud en regard de chaque épanouissement polaire correspondant à chaque extrémité de la bobine nécessite que celle - ci soit alimentée alternativement dans un sens et dans l'autre. Son 
alimentation prend donc la forme d'impulsions alternées, comme indiqué sur le schéma de la figure 7 ci-dessous.

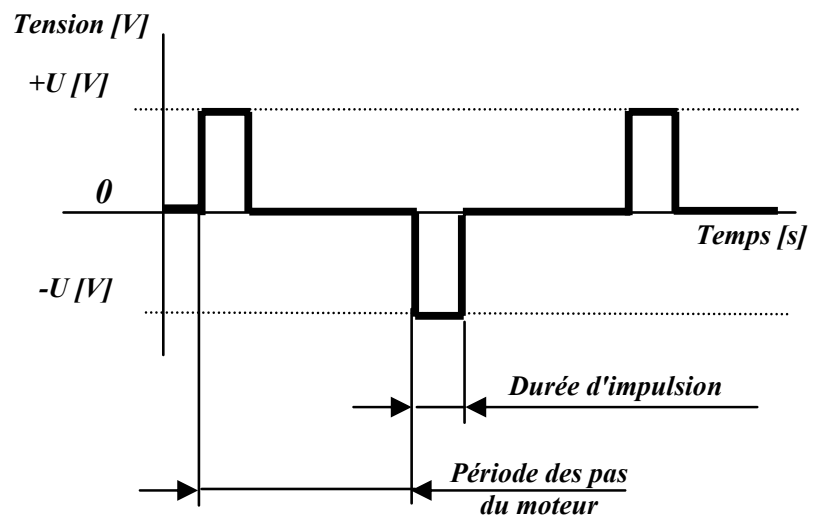

Figure 7. Forme des signaux d'alimentation

4.3.2 Réduction de la consommation Une astuce fondamentale de ce moteur est que l'on profite de l'inertie du rotor pour raccourcir au maximum la durée des impulsions, et, par voie de conséquence la consommation. Les impulsions doivent cependant être suffisamment longues pour que le rotor puisse acquérir l'accélération nécessaire pour vaincre le couple dû aux forces de rappel, quitter sa position d'équilibre pour atteindre la suivante. On définit ainsi un point de fonctionnement dont les paramètres sont la tension appliquée et la longueur des impulsions.

La fréquence des impulsions de commande et, par conséquent la vitesse de rotation du moteur est limitée par les oscillations du rotor autour de sa position d'équilibre. Ce phénomène, n'est en général pas important lorsqu'il s'agit d'une montre où la fréquence est de $1 \mathrm{~Hz}$. Par contre, dans le cas de la capsule, il était impératif de déterminer la vitesse maximum possible pour un fonctionnement sans pertes de pas.

4.3.3 Résultats obtenus Le module réalisé s'inscrit dans un diamètre de $9.5 \mathrm{~mm}$ et son épaisseur totale est de $3.4 \mathrm{~mm}$. Le rapport de réduction de 1200 donne un couple utile sur l'arbre de sortie d'environ $350 \mu \mathrm{Nm}$, suffisant pour assurer le déclenchement de toutes les fonctions. La fréquence maximum des pas, légèrement supérieure à $100 \mathrm{~Hz}$, autorise en sortie une vitesse de rotation de $2.6 \mathrm{t} / \mathrm{mn}$ et des durées de déclenchement des fonctions d'environ 5 secondes. A cette vitesse la consommation est de 500 à $600 \mu \mathrm{A}$.

4.3.4 Conclusion La solution du moto réducteur est intéressante par sa consommation et sa technique de fabrication très éprouvée (figure 8.)

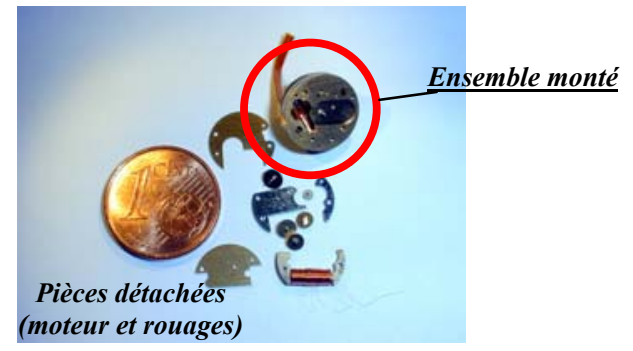

Figure 8. Ensemble moto réducteur
Elle est d'autre part facilement transférable aux entreprises horlogères qui possèdent tous les équipements nécessaires et un savoir-faire permettant de générer des coûts de fabrication très intéressants.

De plus, aucune électronique spécifique n'est nécessaire : les extrémités de la bobine du moteur Lavet sont directement reliées à deux sorties du micro contrôleur

\section{Choix de l'électronique}

\subsection{Description de l'ensemble}

Une liaison haute fréquence, à une distance maximum de quatre à cinq mètres, permet au médecin de surveiller la position de la capsule dans le corps du patient sur un terminal informatique, puis de déclencher au moment voulu les fonctions prévues au montage de cette dernière. (voir schéma fonctionnel de l'ensemble figure 9)

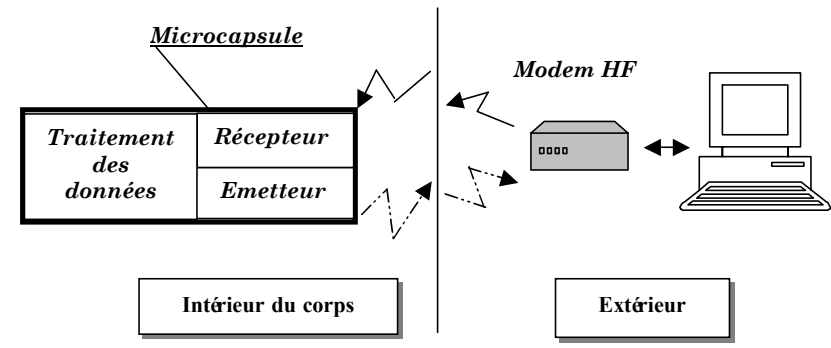

Figure 9. Synoptique de l'ensemble dialogue PC-capsule

\subsection{Réduction de la consommation}

La capacité des piles (50 $\mathrm{mAh})$ et l'autonomie exigée de 24 heures a nécessité un effort important sur la réduction de la consommation de chaque composant: micro contrôleur, émetteur-récepteur embarqué, mais également choix des fréquences, qui doivent de plus être conformes aux fréquences autorisées dans les différentes parties du monde.

5.2.1 Micro contrôleur. Il s'agit d'un micro contrôleur horloger masqué fonctionnant à $32 \mathrm{kHz}$, utilisé en horlogerie. Il a pour avantage de ne consommer que $1 \mu \mathrm{A}$. Le revers de la médaille est que sa faible vitesse limite ses performances, notamment dans la gestion de protocole de transmission des données.

5.2.2 Choix des fréquences. L'utilisation des fréquences radio pose de nombreux problèmes techniques et légaux. Il est obligatoire d'utiliser des fréquences autorisées et compatibles avec l'utilisation. Les bandes ISM (Industrielles, Scientifiques et Médicales) sont réservées à ce type d'utilisation et sont différentes selon les pays. La fréquence doit être conforme à toutes ces législations, en évitant si possible des adaptations par un changement de composants. Elle doit par ailleurs être suffisamment basse pour traverser les tissus sans être trop atténuée. Comme, légalement, elle doit être très stable, une trop basse fréquence nécessiterait l'emploi d'oscillateurs à quartz de taille difficile à avaler. Un compromis dimensions du quartz de l'oscillateur - consommation a été trouvé côté capsule. Dans le sens capsule - PC, la fréquence est de 13.56 MHz. Même si ce n'est pas une valeur idéale pour 
traverser les tissus humains, la réception côté PC a été être optimisée, notamment par l'utilisation d'antennes à gain élevé.

Dans le sens PC - capsule, c'est l'utilisation de la bande $\mathrm{HF}$ de $433 \mathrm{MHz}$, reconnue internationalement pour l'utilisation des tags RF (transfert d'information sans contact) qui a été retenue et qui permet un transfert d'énergie suffisant pour la portée requise.

5.2.3 Choix des protocoles de transmission. Comme leur nom l'indique, les bandes de fréquences ISM sont utilisées pour des applications allant des appareils chirurgicaux aux fours à micro ondes, en passant par les télécommandes de portes de garage.

La fréquence retenue pour la capsule est quasi mondiale. Son utilisation massive dans toutes sortes d'applications implique de prévoir un protocole de transmission sécurisé pour rejeter les parasites. Dans un même temps il doit être suffisamment simple pour ne pas nécessiter une utilisation trop importante des ressources et ainsi limiter la consommation d'énergie. Le protocole réalisé, relativement lent, permet une correction assez simple des erreurs dues au choix de la fréquence.

5.2.4 Emetteur-récepteur embarqué. Une puissance réduite, de l'ordre du $\mathrm{mW}$, garantit la portée d'émission requise, une bonne stabilité et la conformité aux normes. Les périodes d'émission - réception, en synchronisme avec le PC. permettent d'abaisser la consommation moyenne à environ $2 \mu \mathrm{A}$ avec des pointes maximum en fonctionnement de $1 \mathrm{~mA}$.

\section{Modules fonctionnels}

L'optimisation de la consommation a nécessité le bilan énergétique de toute la chaîne cinématique et les éléments situés en aval ne doivent pas offrir de couples ou de forces résistants trop élevés, tout en déployant les forces nécessaires à l'accomplissement des tâches pour lesquelles ils ont été conçus. Ainsi le dispositif moteur est utilisé comme un système de déclenchement qui libère l'énergie emmagasinée dans des éléments élastiques ou par des différences de pression entre l'intérieur et l'extérieur de la capsule. Les substances liquides, par exemples, sont expulsées par l'élasticité de membranes de silicone qui jouent le rôle de réservoir (figure 10).

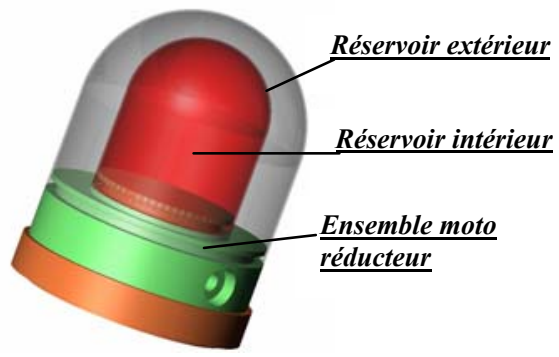

Figure 10. Module de largage de deux liquides

C'est la société Statice Santé, de Besançon, qui a bien voulu nous conseiller dans le dimensionnement et la mise au point de toutes les pièces de silicone contenues dans les modules fonctionnels de la capsule.

Dans le cas du prélèvement de contenu intestinal, c'est une dépression qui assure la fonction (figure 11)

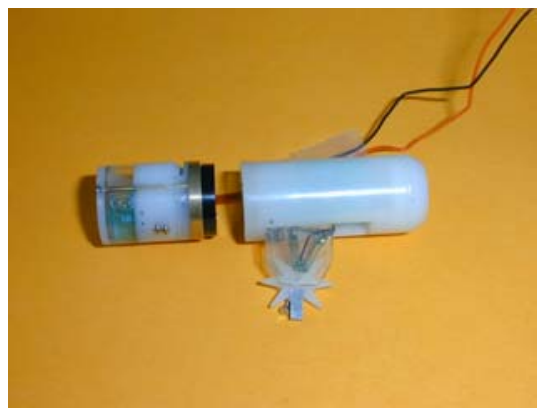

Figure 11Module pour trois prélèvements de contenu intestinal

\section{Conclusion}

La photographie de la figure 12 rassemble, autour de la capsule de Strasbourg, les prototypes réalisés dans le cadre de ce projet: largage de deux liquides, largage de trois poudres ou substances solides, module de trois prélèvements de contenu intestinal. Il faut noter que la conception de ces modules autorise un nombre plus élevé de largages et de prélèvements.

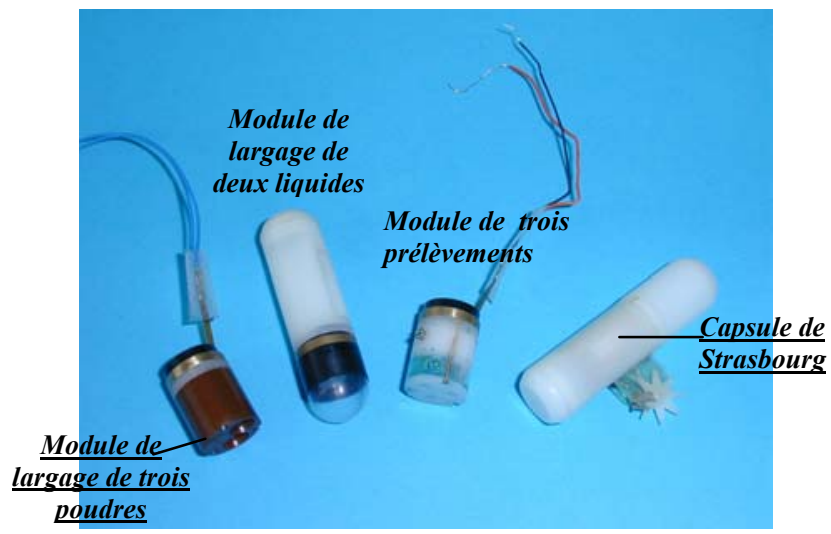

Figure 12. Ensemble des prototypes réalisés

Par le nombre important de fonctions possibles et par leur répétitivité, par son système de localisation simple et précis, son autonomie, ses temps de réponse, ce dispositif se situe en bonne complémentarité avec les autres capsules réalisée dans le monde. Il obéit à tous les objectifs fixés par le cahier des charges. Sa complète modularité au niveau du système de déclenchement, des connexions, du protocoles de transmission des données quels que soient les modules fonctionnels montés et l'utilisation de techniques éprouvées contribuent à le rendre compétitif.

\section{Références}

[1] A. Lambert, F. Vaxman, F. Crenner, T. Wittmann, J.F. Grenier, Medical \& Biological Engineering \& Computing, mars 1991, pp 191-196

[2] La Recherche, avril 1999, p. 22

[3] Ridha Loutil, Industrie et Technologies $n^{\circ} 845$, février 2003, p. 11 